\title{
Computerized Tomography-Guided Paracentesis: An Effective Alternative to Bedside Paracentesis?
}

\author{
Vinaya Gaduputia , Hassan Tariq ${ }^{\mathrm{a}, \mathrm{b}}$, Chaitanya Chandrala ${ }^{\mathrm{a}}$, Sailaja Sakam ${ }^{\mathrm{a}}$, \\ Naeem Abbas ${ }^{\text {a }}$, Sridhar Chilimuria
}

\begin{abstract}
Background: Ascites remains the most common cause of hospitalization among patients with decompensated cirrhosis. Paracentesis is a relatively safe procedure with low complication rates. Computerized tomography (CT)-guided therapeutic paracentesis could be a safe and effective alternative to unaided or aided (ultrasonogram-guided) bedside paracentesis. In this retrospective study, we aimed to compare the efficacy, safety, and cost-effectiveness of CT-guided paracentesis with bedside paracentesis.
\end{abstract}

Methods: The period of study was from 2002 to 2012. All patients with cirrhosis who underwent therapeutic paracentesis were included in the study. These patients were divided into two groups. Group I consisted of patients who underwent CT-guided pigtail catheter insertion with ascitic fluid drainage. Group II consisted of patients who underwent beside therapeutic paracentesis after localization of fluid either by physical examination or sonographic localization. We measured the efficacy of CT-guided paracentesis and bedside paracentesis in terms of volume of fluid removed, length of stay, discharge doses of diuretics (spironolactone and furosemide) and number of days to readmission for symptomatic ascites. We also computed the costeffectiveness of CT-guided therapeutic paracentesis when compared to a bedside procedure. Fischer exact test was used to analyze the distribution of categorical data and unpaired $t$-test was used for comparison of means.

Results: There were a total of 546 unique patients with diagnosed cirrhosis who were admitted to the hospital with symptomatic ascites and underwent therapeutic paracentesis. Two hundred and fortyseven patients underwent CT-guided paracentesis, while 272 patients underwent bedside paracentesis. There was significant inverse correlation between the amount of ascitic fluid removed and total length of stay in the hospital. We found that the volume of fluid removed via a CT-guided pigtail insertion and drainage $(2.72 \pm 2.02 \mathrm{~L})$ is signifi-

Manuscript accepted for publication November 28, 2016

aDepartment of Medicine, Bronx Lebanon Hospital Center, 1650 Selwyn Ave., Suite \#10C, Bronx, NY 10457, USA

${ }^{b}$ Corresponding Author: Hassan Tariq, Department of Medicine, Bronx Lebanon Hospital Center, 1650 Selwyn Ave., Suite \#10C, Bronx, NY 10457, USA. Email: HTARIQ@bronxleb.org

doi: https://doi.org/10.14740/jocmr2832w cantly higher when compared to fluid removed via bedside paracentesis $(1.94 \pm 1.69)$. We also found that the interval time period between two successive therapeutic paracenteses was significantly longer for CT group (106.56 \pm 75.2 days) when compared to the bedside group (25.57 \pm 7.68 days).

Conclusion: CT-guided paracentesis with pigtail catheter insertion and drainage is a clinically effective, cheap and safe alternative to conventional bedside paracentesis.

Keywords: CT-guided bedside paracentesis; Complications of paracentesis; Ascites in cirrhosis; Management of ascites; Therapeutic paracentesis; CT-guided paracentesis; Bedside paracentesis

\section{Introduction}

Ascites is the most common complication of end-stage liver disease with almost half of all patients developing it within 10 years of diagnosis [1]. Cirrhosis is the most common cause of ascites in the USA with almost $85 \%$ of cases attributable to it [2]. The advancements in understanding of mechanics of development of ascites and availability of effective diuretics have led to a decrease in the number of admissions to hospital with symptomatic ascites over the past few decades. However, ascites remains the most common cause of hospitalization among patients with decompensated cirrhosis [3] and therefore has profound implications on quality of life and health care costs. Paracentesis is the most important and basic step in diagnosing the cause of ascites. It has been reported in the literature that paracentesis whether it is of a diagnostic or therapeutic intent, is a relatively safe procedure with low complication rates [4].

While dietary salt restriction and diuretic therapy remains the mainstay for therapy in symptomatic large volume ascites, large volume paracentesis is an effective supplemental modality, especially in cases of diuretic resistant and diuretic intractable cases. It has been demonstrated in some studies that serial large volume paracenteses with concomitant albumin infusion were safer and shortened the duration of hospitalization compared with diuretic therapy alone, in patients with symptomatic ascites [5]. It also of note that single total paracentesis with appropriate colloid replacement was found to be a safer alternative to serial paracenteses [6]. There are multiple modali- 
ties with which a therapeutic paracentesis could be achieved. These include bedside manual or radiology (ultrasound or computerized tomography (CT)) aided ascitic fluid localization with catheter insertion and drainage. Irrespective of the method of paracentesis, it has been opined that an indwelling catheter is not ideally suited for a cirrhotic patient in view of increased susceptibility for infection [7]. However, no studies have been done previously to demonstrated efficacy and safety of CT-guided paracentesis with placement of short-term indwelling drainage catheters, in patients admitted to the hospital with symptomatic ascites.

In this retrospective study, we aimed to compare the efficacy (in terms of volume of fluid removed, length of hospitalization, paracentesis-free interval and percentage of patients undergoing repeat paracentesis within 1 year), safety (rates of bleeding, infection, acute renal failure, bowel perforation, and death), and cost-effectiveness of CT-guided paracentesis with bedside paracentesis.

\section{Materials and Methods}

\section{Patients}

This retrospective study was performed according to the Declaration of Helsinki. The Institution Review Board (IRB) at the study location approved the protocol. The period of study was 10 years from 2002 to 2012 . The data were collected from the electronic medical records of the patients and tabulated in Microsoft Excel. All patients with cirrhosis who underwent therapeutic paracentesis were included in the study. All these patients were admitted to the hospital with symptomatic ascites irrespective of their diuretic resistance or sensitivity. These patients were divided into two groups. Group I consisted of patients who underwent CT-guided pigtail catheter insertion with ascitic fluid drainage. Group II consisted of patients who underwent beside therapeutic paracentesis after localization of fluid either by physical examination or sonographic localization.

We collected the baseline demographic data for both the groups including age, gender and ethnicity. We calculated the model for end-stage liver disease (MELD) scores for both the groups at the time of the procedure. We also looked at usage of antiplatelet medications, lactulose, in both the groups at the time of the procedure.

\section{CT-guided therapeutic paracentesis}

A CT scan of the abdomen was performed for quantification of ascites. The area of proposed catheter insertion was sterilized and $3-5 \mathrm{~mL}$ of $1 \%$ lidocaine was infiltrated down to the peritoneum with a 25 - to 27 -gauge needle. A small dermatomy was made to facilitate the catheter placement. Under direct CT guidance, a paracentesis introducer needle was advanced into the ascitic fluid while aspirating. The drainage catheter was then advanced and deployed to provide access to the peritoneal cavity, while the introducer needle was removed. The catheter was an occlusion resistant, silicone-coated pigtail catheter of
6 or 8 Fr caliber. The catheter was connected to a fluid collection bag and the ascitic fluid was drained in small volumes at frequent intervals over the subsequent few days. The catheter was removed by an interventional radiologist upon satisfactory resolution of ascites.

\section{Bedside paracentesis}

Ascitic fluid was localized using standard physical examination technique of percussion to elicit dullness. The proposed needle entry site was sterilized using povidone iodine solution applied in widening circular motions. The site of insertion was anesthetized using a Z-track technique with $3-5 \mathrm{~mL}$ of $1 \%$ lidocaine administered via a 25 - to 27 -gauge needle. A singlehole, two-piece metal needle (15- or 16-gauge diameters) was then introduced, also in a Z-track technique, into the peritoneal cavity while aspirating simultaneously. Once free flow of ascitic fluid was ascertained, the needle was connected to a fluid collection bag. The ascitic fluid was removed in a single sitting and the needle was removed from the peritoneal cavity.

\section{Evaluation of results}

We measured the efficacy of CT-guided paracentesis and bedside paracentesis in terms of volume of fluid removed, length of stay, discharge doses of diuretics (spironolactone and furosemide) and number of days to readmission for symptomatic ascites. We looked at the safety profile of these two paracenteses in terms of incidence of complications such as leak of ascitic fluid, bleeding and secondary infection of ascitic fluid. We also computed the cost-effectiveness of CT-guided therapeutic paracentesis when compared to a bedside procedure.

\section{Statistical analysis}

Continuous variables were stated as mean and standard deviation and categorical variables as percentages. Fischer exact test was used to analyze the distribution of categorical data and unpaired $t$-test was used for comparison of means. A P-value $<$ 0.05 was considered statistically significant.

\section{Results}

There were a total of 546 unique patients with diagnosed cirrhosis who were admitted to the hospital with symptomatic ascites and underwent therapeutic paracentesis within the study period from January 2002 to December 2012. Out of the study population, 247 patients underwent CT-guided paracentesis while 272 patients underwent bedside paracentesis. We tabulated results only for the first admission of each unique patient.

The baseline characteristics of both the groups are shown in Table 1. We found that all the baseline characteristics were similar among these two groups barring sex distribution (significantly higher percent of males in the bedside group; $\mathrm{P}=$ 
Table 1. Baseline Characteristics of CT-Guided Paracentesis Group and Bedside Paracentesis Group

\begin{tabular}{|llll}
\hline Characteristic & Bedside paracentesis & CT-guided paracentesis & P value \\
\hline $\begin{array}{l}\text { Age (years), mean } \pm \text { SD } \\
\text { Race }\end{array}$ & $57.45 \pm 10.11$ & $58 \pm 11$ & 0.55 \\
$\quad$ African American & $126(46.3)$ & $112(45.3)$ & 0.86 \\
$\quad$ Hispanics & $144(52.9)$ & $134(54.2)$ & 0.79 \\
$\quad$ Others & $2(0.73)$ & $1(0.4)$ & 1 \\
Male sex, no. $(\%)$ & $195(71.7)$ & $137(55.3)$ & $0.0002^{\ddagger}$ \\
MELD score, mean \pm SD & $18.79 \pm 7.55$ & $20.99 \pm 7.25$ & $0.0006^{\ddagger}$ \\
Medications, no. $(\%)$ & & & 0.31 \\
$\quad$ Lactulose & $196(72)$ & $188(76)$ & 0.79 \\
$\quad$ NSBB & $146(53.7)$ & $136(57.02)$ & 0.62 \\
Aspirin & $82(30.1)$ & $69(27.9)$ & 0.26 \\
\hline Plavix & $9(0.03)$ & $4(0.016)$ & \\
\hline
\end{tabular}

${ }^{\dagger}$ MELD scoring is a system for assessing the severity of chronic liver disease and is calculated by the formula: MELD = 3.78(Ln serum bilirubin $(\mathrm{mg} / \mathrm{dL}))+11.2(\mathrm{Ln} I \mathrm{NR})+9.57(\mathrm{Ln}$ serum creatinine $(\mathrm{mg} / \mathrm{dL}))+6.43$. ${ }^{\mathrm{T}} \mathrm{NSBB}$ : non-selective beta blockers. ${ }^{\mathrm{A}}<0.05$ was considered statistically significant.

0.0002) and mean MELD score (significantly higher in the CT group; $\mathrm{P}=0.0006)$.

Outcome measures: volume of fluid removed, length of stay, and interval between two successive therapeutic paracenteses

We observed that there was a significant inverse correlation between the amount of ascitic fluid removed and total length of stay in the hospital ( $\mathrm{P}<0.0001 ; 95 \%$ CI of slope: -0.001398 to $\left.-0.0007311 ; \mathrm{R}^{2}=0.07076\right)$, in patients admitted with symptomatic ascites (Fig. 1). Table 2 demonstrates the outcomes in $\mathrm{CT}$ and bedside paracentesis groups. We found that the volume fluid removed via a CT-guided pigtail insertion and drainage $(3.54 \pm 2.61 \mathrm{~L})$ is statistically significantly higher $(\mathrm{P}$ $<0.0001$ ) when compared to fluid removed via bedside paracentesis $(2.15 \pm 2.06)$. As expected from our linear regression analysis, the higher volume of ascitic fluid removed translated into shorter length of hospital stay within the CT-guided paracentesis group $(10.49 \pm 9.97$ days versus $12.24 \pm 8.54$ days in bedside group with a $\mathrm{P}=0.0318$ ). We also found that the interval time period between two successive therapeutic paracenteses was significantly longer $(\mathrm{P}=0.0032)$ for CT group $(53.88$ \pm 50.03 days) when compared to the bedside group (38.69 \pm 33.72 days). However, we did not find a significant difference $(\mathrm{P}=0.93)$ between the percentages of patients requiring a therapeutic paracentesis within 1 year among CT group (52.6\%) and bedside group (52.9\%)

\section{Safety}

We looked at all the major complications such as bleeding, infection, bowel perforation and death attributable to procedure, in either of the groups. Bleeding complication was defined as a drop in hemoglobin levels by $1 \mathrm{~g} / \mathrm{dL}$ or occurrence of hematomas. There were four such bleeding complications in the bedside group $(1.4 \%)$ with two of them being rectus muscle hematomas, while there were none among the CT group. However, none of these four patients required packed red blood cell (PRBC) transfusions. The pigtail catheter that was inserted via CT guidance was left behind for drainage for an average of 1.56 days. We looked at the infection rates among either of these groups. Positive bacterial cultures from the first ascitic fluid samples that were sent during the index admission were attributed to be secondary to spontaneous bacterial peritonitis and were not included in the calculations. A positive bacterial culture from second specimen of ascitic fluid that was sent during the same index admission was considered as secondary bacterial peritonitis and was attributed to the preceding paracentesis procedure. There were four such positive cultures in the CT group (each growing Staphylococcus hominis, methi-

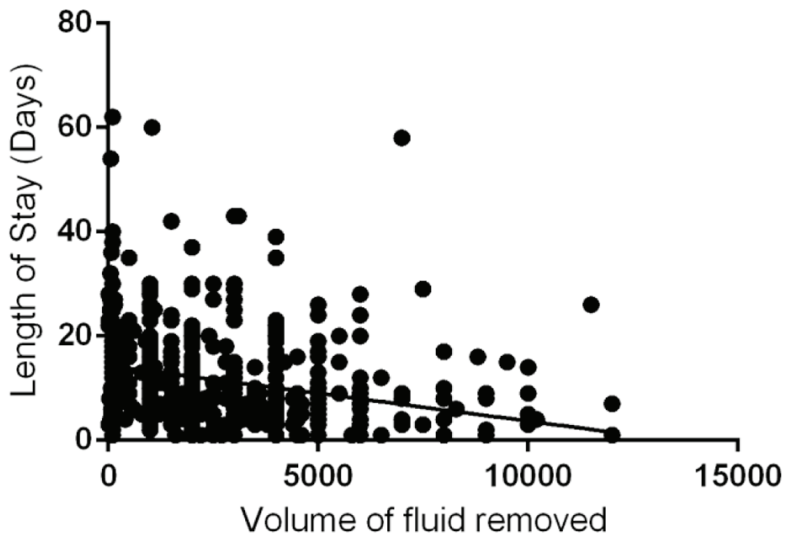

Figure 1. Correlation between volume of ascetic fluid $(\mathrm{mL})$ and hospital length of stay $(\mathrm{P}<0.0001 ; 95 \% \mathrm{Cl}$ of slope -0.001398 to -0.0007311 ; $\left.R^{2}=0.07076\right)$. 
Table 2. Outcomes in CT-Guided Paracentesis Group and Bedside Paracentesis Group

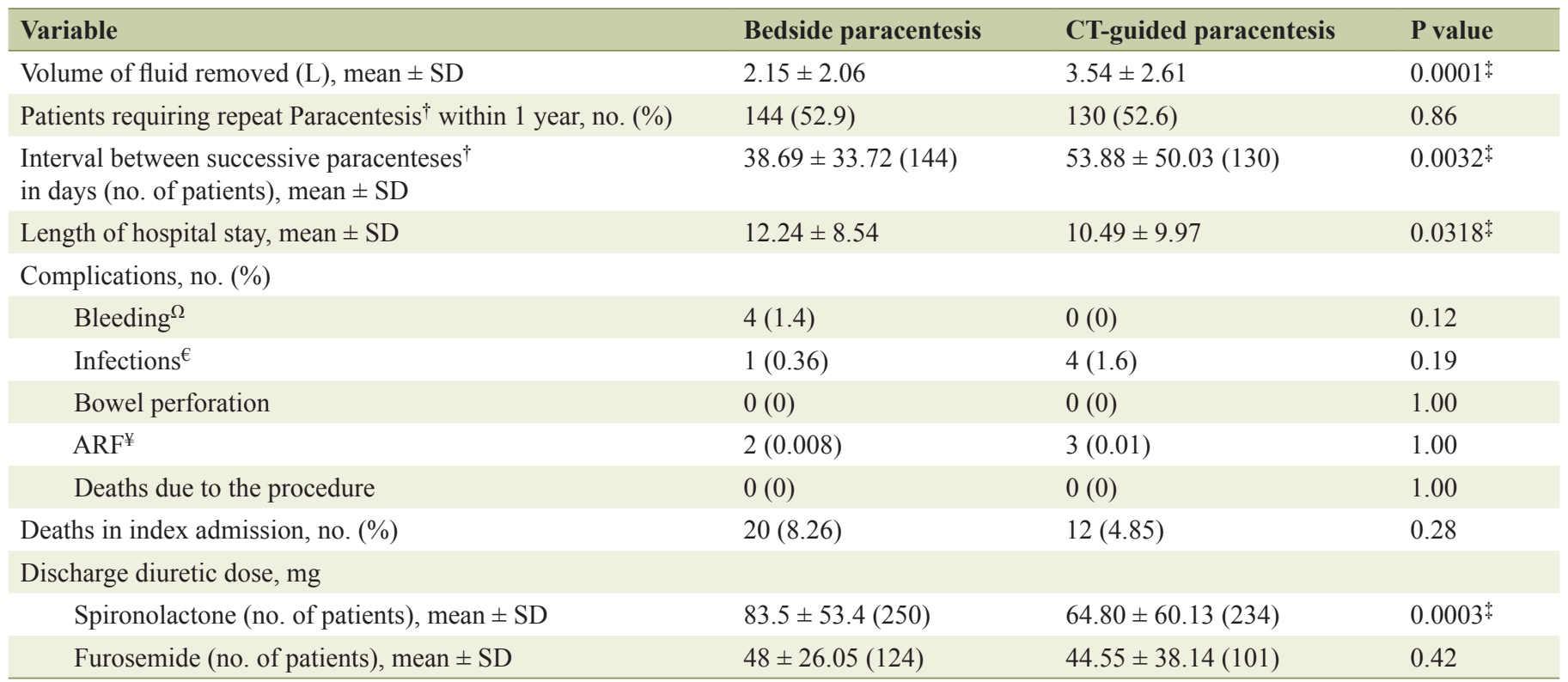

${ }^{\dagger}$ Therapeutic paracenteses only. Diagnostic paracenteses were excluded. ${ }^{\dagger} \mathrm{P}<0.05$ was considered statistically significant. ${ }^{\Omega}$ Defined as drop in

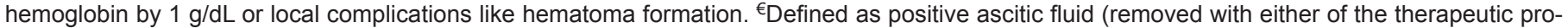
cedures) bacterial cultures after an initial negative culture result during same admission (thus ruling out SBP). ${ }^{*}$ Defined as absolute increase of $\geq 0.3$ $\mathrm{mg} / \mathrm{dL}$ in serum creatinine concentration or $\mathrm{a} \geq 50 \%$ increase in the serum creatinine concentration within $48 \mathrm{~h}$ of procedure.

cillin-sensitive Staphylococcus aureus, Klebsiella pneumoniae and Escherichia coli, respectively) when compared to only one positive culture in the bedside group (culture grew Escherichia coli). However, this difference was not statistically significant $(\mathrm{P}=0.19)$. Acute renal failure $(\mathrm{ARF})$ is a known complication of large volume paracentesis. There were two cases of ARF in the bedside paracentesis group and three cases of ARF in the CT group. There was no statistical difference in the incidence of ARF between the two groups $(\mathrm{P}=1.00)$. We did not find a statistically significant relation between the number of days that the catheter remained within the peritoneal cavity and incidence of these secondary infections $(P=0.459)$. There were no documented bowel perforations or deaths attributable to the therapeutic paracenteses in either group.

\section{Discharge doses of diuretics}

The discharge doses of diuretics were also compared between the two groups. Spironolactone was prescribed to every patient who had no contraindications (severe hyperkalemia and worsening renal function; two had contraindications in bedside group and one in CT group) at the time of discharge (the deaths during the index hospitalization were excluded). The mean discharge dose of spironolactone in the CT group (64.80 $\pm 60.13 \mathrm{mg})$ was significantly less $(\mathrm{P}=0.0003)$ than that of the bedside group $(83.5 \pm 53.4 \mathrm{mg})$. There was no statistical difference $(\mathrm{P}=0.28)$ between the percentages of discharged patients who were on furosemide, among the bedside $(45.5 \%)$ and CT groups (40.8\%). Furosemide was prescribed in 124 patients in bedside group (mean dose of $48 \pm 26.05 \mathrm{mg}$ ) and 101 patients in CT group (mean dose of $44.55 \pm 38.14$ ), respectively, with no statistical difference in the average doses between these two groups $(\mathrm{P}=0.42)$.

\section{Discussion}

Portal hypertension (sinusoidal) is crucial for development of ascites in patients with cirrhosis $[8,9]$. Portal pressure that is required for triggering retention of fluid is generally accepted to be $\geq 12 \mathrm{~mm} \mathrm{Hg}$ [8]. Rate of ascites formation is a direct function of portal pressure. Ascites is typically treated in initial stages with a non-invasive strategy encompassing a combination between strict sodium restriction and diuretics. However, refractory ascites often requires invasive measures such as repeated therapeutic paracenteses or placement of TIPS. We found with logistic regression analysis that the length of stay for patients admitted to the hospital with symptomatic ascites requiring therapeutic paracentesis is inversely related to the amount of fluid removed with paracentesis. We found that the severity of liver disease was more in the CT group when compared to the bedside group, measured in terms of the mean MELD scores. The higher MELD score and therefore more severe liver disease in CT group notwithstanding, the outcomes including volume of fluid removed with a single procedure, length of stay, and interval between successive paracenteses were significantly better in the CT group. The mean discharge dose of spironolactone was significantly less in the CT group when compared to the bedside group, which could be a reflection of more optimal fluid status in a patient who underwent CT-guided paracentesis. 
The average Medicare reimbursement rate for a CT-guided paracentesis procedure, in between 2002 and 2012, was about $650 \$$. The average daily inpatient cost, based on average Medicare reimbursement rates in between 2002 and 2012, involved in the management of patients with decompensated cirrhosis requiring bedside paracentesis was $800 \$ /$ day. We found that the significantly shorter length of stay in the CT group (1.75 days) when compared to the bedside group, offset any costs of the radiological procedure.

The incidence of hemorrhagic complications even in the setting of coagulation abnormalities such as those seen in patients with cirrhosis and risk of infection was demonstrated to be low [4]. There has been no demonstrated correlation between degree of coagulopathy or thrombocytopenia and risk of hemorrhagic complications with paracentesis in patients with cirrhosis [10]. The studies demonstrated these low rates of bleeding complications employed usage of narrow metal needles to tap ascitic fluid from areas of percussed dullness or ultrasonographic localization. It has been recommended to use 15- or 16-gauge needles (outer diameter ranging from 1.65 to $1.82 \mathrm{~mm}$ ) for therapeutic paracentesis [11]. It has been noted in older studies that usage of larger caliber trocars led to more serious hemorrhagic complications $[12,13]$. With advancements and greater availability of interventional radiographic techniques, CT-guided therapeutic paracentesis could be a safe and effective alternative to unaided or aided (ultrasonogramguided) bedside paracentesis. The usual caliber of catheter inserted for ascitic fluid drainage ranges in between 6 and 8 French (outer diameter ranging from 2 to $2.7 \mathrm{~mm}$ ). Consistent with these observations, we found that no one among the CT group had any bleeding complications.

In theory, the ascites associated with cirrhosis is more prone to infection due to decreased opsonic activity within the ascitic fluid [14]. Other factors which could explain the increased susceptibility to infections in these patients include existence of a pervasive acquired immune deficiency in cirrhosis [15], serum complement deficiency [16], and dysfunctional neutrophils/stationary phagocytes $[17,18]$. However, in spite of these concerns, we did not find any untoward events, especially infections, occurring in a statistically significant higher frequency among the patients who underwent CT-guided pigtail catheter insertion and drainage of ascitic fluid when compared to bedside paracentesis. We found that the length of peritoneal catheterization after CT-guided insertion/drainage was not related to the incidence of infections. However, the very low incidence rate of infection (1.6\%) might have led to a paucity of statistical power to show any significant difference.

It has been described that the right lower quadrant is a suboptimal choice for paracentesis in view of higher risk of bowel perforation, especially in patient on lactulose who are known to have distended ceca [19]. Yet, right lower quadrant bedside paracenteses are routinely performed. Theoretically, a CT-guided procedure would eliminate the risk of bowel penetration. It could also be argued that bedside paracentesis could get more technically challenging in view of increasing abdominal obesity rates, thus making manual localization of ascitic fluid difficult.

Our study was beset by disadvantages inherent to a retrospective study. Even though we took only patients with primary admitting diagnosis of symptomatic ascites requiring therapeutic paracentesis, multiple unrecognized confounding variables could have led to difference in lengths of hospitalization between the two groups with different methods of paracentesis. Even though we found that the CT group had a significantly higher MELD score and therefore technically more severe liver disease, an extrapolation that the rate of recurrence of ascites will be higher in the CT group cannot be made. We did not measure portal pressures, which could have given a more accurate measure of rate of recurrence of ascites. The contributions of other complications of advanced liver disease such as hepatic encephalopathy and variceal bleeding towards the length of hospitalization could not be individually ascertained. The amount of fluid removed by bedside paracentesis is subject to significant inter-technician variability depending on level of expertise (attendings, fellows, residents or interns), which we assumed, might have been offset by the high number of patients and long duration of study (10 years) involving different sets of physicians. Another drawback of the study was that we did not include the admission doses of diuretics as it was not possible for us to confirm the patient compliance at home. We did not have urine electrolyte panels on each patient to evaluate for medication compliance, dietary compliance and renal diuretic sensitivity/resistance. It could also be argued that patients who underwent bedside paracentesis were otherwise much sicker (not considering MELD score and intrinsic liver disease) due to other medical co-morbidities, thus precluding them from being transported to radiology department for the CT-guided procedure. This selection bias could therefore explain the differences in hospitalization rates among these groups.

In conclusion, we found that CT-guided paracentesis with pigtail catheter insertion and drainage is a clinically effective, cheap and safe alternative to conventional bedside paracentesis. However, in view of limitations of this retrospective study, prospective studies need to be done to establish the safety and efficacy of CT-guided paracentesis.

\section{Competing Interests}

The authors declare that they have no competing interests.

\section{Disclosures}

None.

\section{Author Contributions}

All authors have made contributions to the article and have reviewed it before submission.

\section{References}

1. Gines P, Quintero E, Arroyo V, Teres J, Bruguera M, Rimola A, Caballeria J, et al. Compensated cirrho- 
sis: natural history and prognostic factors. Hepatology. 1987;7(1):122-128.

2. Runyon BA. Management of adult patients with ascites due to cirrhosis: an update. Hepatology. 2009;49(6):20872107.

3. Lucena MI, Andrade RJ, Tognoni G, Hidalgo R, De La Cuesta FS. Multicenter hospital study on prescribing patterns for prophylaxis and treatment of complications of cirrhosis. Eur J Clin Pharmacol. 2002;58(6):435-440.

4. Runyon BA. Paracentesis of ascitic fluid. A safe procedure. Arch Intern Med. 1986;146(11):2259-2261.

5. Quintero E, Gines P, Arroyo V, Rimola A, Bory F, Planas $\mathrm{R}$, Viver $\mathrm{J}$, et al. Paracentesis versus diuretics in the treatment of cirrhotics with tense ascites. Lancet. 1985;1(8429):611-612.

6. Tito L, Gines P, Arroyo V, Planas R, Panes J, Rimola A, Llach J, et al. Total paracentesis associated with intravenous albumin management of patients with cirrhosis and ascites. Gastroenterology. 1990;98(1):146-151.

7. Moore KP, Aithal GP. Guidelines on the management of ascites in cirrhosis. Gut. 2006;55(Suppl 6):vi1-12.

8. Gines P, Fernandez-Esparrach G, Arroyo V, Rodes J. Pathogenesis of ascites in cirrhosis. Semin Liver Dis. 1997; 17(3):175-189.

9. Morali GA, Sniderman KW, Deitel KM, Tobe S, WittSullivan H, Simon M, Heathcote J, et al. Is sinusoidal portal hypertension a necessary factor for the development of hepatic ascites? J Hepatol. 1992;16(1-2):249-250.

10. Webster ST, Brown KL, Lucey MR, Nostrant TT. Hemorrhagic complications of large volume abdominal paracen- tesis. Am J Gastroenterol. 1996;91(2):366-368.

11. Runyon BA. Introduction to the revised American Association for the Study of Liver Diseases Practice Guideline management of adult patients with ascites due to cirrhosis 2012. Hepatology. 2013;57(4):1651-1653.

12. Mallory A, Schaefer JW. Complications of diagnostic paracentesis in patients with liver disease. JAMA. 1978;239(7):628-630.

13. Liebowitz HR. Hazards of abdominal paracentesis in the cirrhotic patient. 2. N Y State J Med. 1962;62:1997-2004.

14. Runyon BA, Morrissey RL, Hoefs JC, Wyle FA. Opsonic activity of human ascitic fluid: a potentially important protective mechanism against spontaneous bacterial peritonitis. Hepatology. 1985;5(4):634-637.

15. Runyon BA. Bacterial infections in patients with cirrhosis. J Hepatol. 1993;18(3):271-272.

16. Runyon BA. Low-protein-concentration ascitic fluid is predisposed to spontaneous bacterial peritonitis. Gastroenterology. 1986;91(6):1343-1346.

17. Rimola A, Soto R, Bory F, Arroyo V, Piera C, Rodes J. Reticuloendothelial system phagocytic activity in cirrhosis and its relation to bacterial infections and prognosis. Hepatology. 1984;4(1):53-58.

18. Laffi G, Carloni V, Baldi E, Rossi ME, Azzari C, Gresele $\mathrm{P}$, Marra F, et al. Impaired superoxide anion, platelet-activating factor, and leukotriene B4 synthesis by neutrophils in cirrhosis. Gastroenterology. 1993;105(1):170-177.

19. Sakai H, Sheer TA, Mendler MH, Runyon BA. Choosing the location for non-image guided abdominal paracentesis. Liver Int. 2005;25(5):984-986. 